\title{
Effect of the form of the sesame-based diet on the absorption of lignans
}

\author{
Emmanouil N. Papadakis ${ }^{1}$, Despina Lazarou ${ }^{2}$, Raphael Grougnet ${ }^{3}$, Prokopios Magiatis ${ }^{3}$, \\ Alexios L. Skaltsounis ${ }^{3}$, Euphemia Papadopoulou-Mourkidou ${ }^{1}$ and Athanasios I. Papadopoulos ${ }^{2 *}$ \\ ${ }^{1}$ Pesticide Science Laboratory, School of Agriculture, Aristotle University of Thessaloniki, PO Box 1678, Thessaloniki 54006, \\ Greece \\ ${ }^{2}$ Animal Physiology Laboratory, School of Biology, Faculty of Science, Aristotle University of Thessaloniki, Thessaloniki 54124, \\ Greece \\ ${ }^{3}$ Division of Pharmacology and Natural Products Chemistry, Department of Pharmacy, University of Athens, Panepistimioupolis \\ Zografou, GR 15771 Athens, Greece
}

(Received 27 July 2007 - Revised 4 March 2008 - Accepted 9 March 2008 - First published online 23 April 2008)

The effect of different forms of sesame-based diets on the concentration of plasma lignans was assayed by estimating the levels of certain lignans (sesame lignans and enterolignans) in the plasma of experimental animals. In a series of experiments, male Wistar rats were fed either a raw sesame-enriched diet or a tahini-enriched diet. The plasma concentration of the lignans (sesame lignans and enterolignans) was determined at various time intervals over a $24 \mathrm{~h}$ period after a single administration. Enterodiol and enterolactone concentration in the tahini-treated group was significantly higher than in the raw sesame-treated group. In another series of experiments, male Wistar rats were fed, for $15 \mathrm{~d}$, diets enriched in raw dehulled sesame, sesame perisperm, sesame oil, tahini and a polyphenolic extract derived from the seed perisperm. Enterodiol and enterolactone plasma concentration was high in the case of the sesame perisperm in spite of its low concentration in the assessed sesame lignans. Overall, the levels of the sesame lignans and enterolignans present in plasma seem to be influenced not only by the amount of lignan intake but also by other factors such as the form of the sesame-based diet.

Sesame: Lignans: Enterolactone: Sesamin

Lignans are diphenolic compounds that contain a 2,3-dibenzylbutane structure formed from the dimerisation of two cinnamic acid residues ${ }^{(1)}$. Lignans are widely distributed among several species of the plant kingdom and their biological role has not yet been fully elucidated ${ }^{(2)}$. Foods known to contain large amounts of lignans are mainly whole-grain products, berries, tea, vegetables of the Brassica genus, flaxseed and sesame $^{(3)}$. In a number of studies, lignan intake has been associated with a reduced risk of certain cancer types as well as other beneficial effects ${ }^{(1,4)}$.

Certain lignans, collectively named mammalian lignans or enterolignans, have been identified as natural constituents of the biological fluids (blood, urine, semen, prostatic fluid) of mammals ${ }^{(2)}$. It has been reported that the consumption of lignan-rich foods results in the increase of the concentration of the enterolignans in the mammalian blood and urine ${ }^{(5,6)}$. According to studies performed in vitro, when the lignans most commonly found in food (secoisolariciresinol, matairesinol, pinoresinol, lariciresinol, sesamin) are incubated with human faecal microflora ${ }^{(7,8)}$, they are transformed to the enterolignans enterodiol and/or enterolactone. Epidemiological research studies have demonstrated that a high serum concentration in enterolactone has been associated with a decreased risk of hormone-related diseases such as prostate and breast cancer ${ }^{(4,9)}$.

In Asian countries the consumption of sesame is high. Sesame oil is used in many dishes and sesame is used for the production of tahini, a paste of roasted sesame seed, and halva, a mixture of tahini and sugars. In Western countries sesame is mainly used as a condiment in biscuits and other bakery products as well as in the preparation of sesame bars.

For the preparation of the sesame-based food products the seed hull (perisperm) is removed. Its disposal is a major concern for the industry and ways for exploitation, such as utilisation in animal feed, are under examination ${ }^{(10)}$. Recently, a polyphenol-rich extract from the sesame perisperm has been obtained $^{(11)}$.

The principal lignans of the sesame seed are sesamin and sesamolin $^{(12)}$. The effect of raw sesame seed consumption as well as isolated sesame lignan administration on various parameters on the physiology of the organism is the subject of numerous investigations ${ }^{(13-16)}$. It has been reported that intake of sesame seeds or isolated lignans results in the presence of sesame lignans in plasma or serum ${ }^{(8,15-18)}$. However, to our knowledge, a comparative study investigating the effect of sesame-based food products, such as tahini, sesame seeds 
and sesame oil, upon the levels of certain lignans in the plasma after a short or a long period of consumption has never been attempted in the past. Industrial processes such as roasting and refining may result in the transformation of sesamin into episesamin and sesamolin into sesaminol and/or sesamol ${ }^{(19)}$ in addition to alterations of the form of the food matrix.

The aim of the present study was to provide data on the effect the form of the sesame-based diet had upon the absorption of the lignans by the organism. For this purpose: (a) we compared the rates of absorption of the lignans between a sesame-based product and raw sesame seeds, and (b) we determined the plasma levels of the lignans after the repeated administration of sesame-based food products, by-products and raw seeds.

\section{Materials and methods}

\section{Chemicals and reagents}

Hexane, acetone, diethyl ether and dichloromethane of analytical grade were from Carlo Erba (Milan, Italy), and methanol and acetonitrile of HPLC grade were purchased from Merck (Darmstadt, Germany). $\beta$-Glucuronidase/sulfatase from Helix pomatia juice was obtained from Sigma Chemicals (St Louis, MO, USA). Lichrolut EN cartridges $(200 \mathrm{mg})$ were also from Merck. Amino-propyl cartridges $(500 \mathrm{mg})$ were purchased from Supelco (Bellefonte, PA, USA). Analytical standards of enterodiol and enterolactone were from Fluka (Buchs, Switzerland). Sesamin, sesaminol, sesamolin, pinoresinol and lariciresinol were isolated as reported elsewhere ${ }^{(11)}$. Stock solutions of the above analytes were made in methanol; working standard solutions containing each analyte at $0.05-5 \mu \mathrm{g} / \mathrm{ml}$ were also prepared in methanol. These solutions were injected into the chromatography system and were used for plotting the calibration curves for each compound. The aforementioned working standard solutions were also used in the preparation of the fortified plasma samples.

\section{Animals and diets}

Diets were prepared by using commercial non-purified feed (type 510K; Hellenic Feed Company, Plati, Greece), raw dehulled sesame seeds, sesame seed perisperm, sesame oil and tahini (Haitoglou SA, Thessaloniki, Greece). The polyphenolic extract was obtained from sesame perisperm as reported elsewhere ${ }^{(11)}$. For short-term feeding $(24 \mathrm{~h})$, raw seeds and tahini were mixed with animal feed to form mixtures of 80 and $65 \%$, respectively. For extended feeding (15d), raw seeds, tahini, seed perisperm, sesame oil and polyphenolic extract were mixed with animal feed to form mixtures of 30,30, 20, 15 and $0 \cdot 1 \%$, respectively (Table 1 ). In both experiments, male Wistar rats obtained from the Medical School of the Aristotle University of Thessaloniki were used. The conduct of animal experimentation was approved by the Regional Veterinary Directorate (licence no. 13/9749/ 12-07-2006).

Experiment 1. Seven-week-old animals (weight 170 (SEM 4.6) g) were housed under controlled conditions, temperature $\left(20-25^{\circ} \mathrm{C}\right)$ and lighting $(12 \mathrm{~h}$ light-dark cycle). Animals were fed on a commercial non-purified diet for 1 week, after which they were randomly divided into six groups of three animals each and fasted for $24 \mathrm{~h}$. On the next day, all groups but one (used as time point 0 ) were provided with a mixture consisting of $80 \%$ raw seeds and $20 \%$ commercial feed. Animals had access to food for $30 \mathrm{~min}$ after which they were transferred to new cages. Animals were anaesthetised using diethyl ether and killed $0,2,4,6,8$ and $24 \mathrm{~h}$ after food withdrawal. The same experiment was repeated using a mixture consisting of $65 \%$ tahini and $35 \%$ commercial feed.

Experiment 2. Seven-week-old male Wistar rats (180 g average body weight) were housed as described above. Animals were fed on a commercial non-purified diet for 1 week after which they were randomly divided into six groups (three animals per group), and fed on either the commercial feed diet or one of the experimental diets described above containing raw seeds, seed perisperm, sesame oil, tahini or polyphenolic extract for $15 \mathrm{~d}$. For each animal, $20 \mathrm{~g}$ food was provided daily. At the end of the experiment, animals were fasted for $12 \mathrm{~h}$ and killed after anaesthetising using diethyl ether.

\section{Determination of plasma lignans}

Blood was collected from each animal and dispensed into heparin-containing tubes. Plasma was obtained by centrifuging blood samples at $2000 \mathrm{rpm}$ for $20 \mathrm{~min}$ at $4^{\circ} \mathrm{C}$, and stored at $-70^{\circ} \mathrm{C}$ until analysed.

Table 1. Composition of the experimental diets $(\mathrm{g} / 100 \mathrm{~g})$

\begin{tabular}{|c|c|c|c|c|c|c|c|c|}
\hline & \multicolumn{2}{|c|}{ Experiment 1} & \multicolumn{6}{|c|}{ Experiment 2} \\
\hline & Seeds* & Tahini† & Controlł & Seeds§ & Tahini\| & Oilq & Perisperm** & Extract†† \\
\hline Protein & $25 \cdot 7$ & $25 \cdot 6$ & $21 \cdot 0$ & $22 \cdot 8$ & $23 \cdot 2$ & $17 \cdot 9$ & $18 \cdot 9$ & 20.9 \\
\hline Carbohydrate & $11 \cdot 1$ & $19 \cdot 5$ & $55 \cdot 6$ & 38.9 & $40 \cdot 9$ & $47 \cdot 3$ & 44.5 & $55 \cdot 2$ \\
\hline Fat & $45 \cdot 8$ & $40 \cdot 3$ & $6 \cdot 2$ & $21 \cdot 1$ & 21.9 & $35 \cdot 3$ & $7 \cdot 4$ & $6 \cdot 1$ \\
\hline Fibre & $12 \cdot 1$ & $11 \cdot 2$ & 4.5 & $7 \cdot 4$ & $7 \cdot 6$ & 3.8 & $12 \cdot 0$ & 4.5 \\
\hline
\end{tabular}

* Seeds: raw dehulled sesame seeds-commercial feed, 80:20.

†Tahini: tahini-commercial feed, 65:35.

$\ddagger$ Control: commercial feed.

§Seeds: raw dehulled sesame seeds-commercial feed, 30:70.

|| Tahini: tahini-commercial feed, 30:70.

1 Oil: sesame oil-commercial feed, 15:85

** Perisperm: sesame seed perisperm-commercial feed, 20:80

†† Extract: polyphenolic extract-commercial feed, 0.1:99.9. 
Plasma samples were thawed and hydrolysed with $\beta$-glucuronidase at $37^{\circ} \mathrm{C}$ for $3 \mathrm{~h}$. The lignans were extracted from the hydrolysed samples by the use of Lichrolut EN cartridges. Clean-up was performed on amino-propyl cartridges. Determination of the lignans was performed on an HPLC system equipped with a diode array detector (Thermo Separation Products, Austin, TX, USA). Chromatographic separation was performed on a Zorbax XDB C-18 (Agilent Technologies, Wilmington, DE, USA) column by employing a gradient program using ammonium acetate buffer $(\mathrm{pH} 4)$ and acetonitrile. Peak identification in the plasma samples was based on the retention times and UV spectra of the reference compounds.

Calibration curves were linear for all compounds $\left(R^{2}>0.99\right)$ in the range of $1-250 \mathrm{ng}$. Recoveries were assayed at three concentration levels $(50,200,1000 \mathrm{ng} / \mathrm{ml})$ and were in the range of $71-108 \%$. Relative standard deviation was lower than $11 \%$ in all cases. Limits of quantification were $4-8 \mathrm{ng} / \mathrm{ml}$.

\section{Determination of sesame lignans in experimental diets}

Portions, $100 \mathrm{mg}$, of each experimental diet were vortexed for $1 \mathrm{~min}$ with $2 \mathrm{ml}$ hexane. After centrifugation, the supernatant fraction was collected and the above procedure was repeated twice. The hexane extracts were stored for processing later on. An amount of $2 \mathrm{ml}$ of an acetone-water (80:20) mixture was added to the residue, and after vortexing $(1 \mathrm{~min})$ and centrifugation the supernatant fraction was collected and the above procedure was repeated two more times. The combined supernatant fractions were evaporated under $\mathrm{N}_{2}$ until acetone was removed. To the aqueous solution that remained, $1 \mathrm{ml}$ sodium acetate buffer $(\mathrm{pH} \mathrm{5)}$ containing $1000 \mathrm{U} \beta$-glucuronidase was added and the mixture was incubated at $37^{\circ} \mathrm{C}$ for $3 \mathrm{~h}$. The lignans were extracted three times with $3 \mathrm{ml}$ portions of diethyl ether and the extracts were added to the hexane extracts, evaporated to dryness, dissolved in $500 \mu \mathrm{l}$ methanol and mixed with $500 \mu \mathrm{l}$ ammonium acetate buffer $(\mathrm{pH} 4)$. Chromatographic determination was carried out as in the case of plasma lignans.

\section{Statistics}

Results are reported as means with their standard errors. Statistical analysis was performed using SPSS (version 15.0; SPSS, Inc., Chicago, IL, USA). The data were analysed using one-way ANOVA after applying logarithmic transformation to all values. Multiple comparison tests were performed by the Student-Newman-Keuls test. A difference was considered to be significant at $P<0 \cdot 05$.

\section{Results}

\section{Animal diets and lignan intake}

The lignan intake for each experimental group of animals was calculated by taking into account the concentration of the lignans in the diets provided and the food intake, and is presented in Table 2. In both series of experiments, sesamin was the most abundant lignan, followed by sesamolin and sesaminol. Care was taken to provide the animals with similar amounts of lignans through the various diets, whenever this was possible. The amount of each sesame-based product used in the preparation of the experimental diets was adjusted so that the final amount of each lignan would be similar for all the five lignans that were determined. However, that was feasible only for the three forms of sesame-based products, i.e. seeds, tahini and oil. The lignan composition of perisperm is significantly different from that of the seed and therefore it is not feasible to achieve a balance between the amounts of individual lignans offered. The same condition also applies for the polyphenolic extract in addition to the limitation of the amount of extract available that was prohibitive for largescale experiments.

\section{Short-term feeding (experiment 1)}

Significant differences were observed, in both the values of the maximum concentration and the time those were recorded, between the two groups of experimental animals. In the plasma of the controls enterolactone only was detected (Fig. 1).

Table 2. Lignan intake in experiments of short-term feeding (experiment 1) and extended feeding (experiment 2)

Intake*

\begin{tabular}{|c|c|c|c|c|c|c|c|c|}
\hline \multirow[b]{2}{*}{ Lignans } & \multicolumn{2}{|c|}{$\begin{array}{l}\text { Experiment } 1 \\
\text { ( } \mu \mathrm{mol} / \text { animal) }\end{array}$} & \multicolumn{6}{|c|}{ Experiment 2 ( $\mu \mathrm{mol} /$ animal per $\mathrm{d})$} \\
\hline & Seeds† & Tahinił & Control§ & Seeds $\|$ & Tahini & $\mathrm{Oil}^{* *}$ & Perisperm†† & Extract $\ddagger$ \\
\hline Lariciresinol & 0.30 & 0.42 & $0 \cdot 13$ & 0.51 & 0.84 & 0.45 & $2 \cdot 57$ & $1 \cdot 25$ \\
\hline Pinoresinol & $1 \cdot 22$ & 1.58 & $0 \cdot 20$ & 2.09 & $3 \cdot 20$ & 0.49 & $4 \cdot 51$ & 1.42 \\
\hline Sesaminol & $3 \cdot 14$ & $5 \cdot 62$ & - & $5 \cdot 20$ & $10 \cdot 97$ & $0 \cdot 21$ & $4 \cdot 50$ & $1 \cdot 14$ \\
\hline Sesamin & $20 \cdot 02$ & 14.95 & - & $35 \cdot 66$ & 30.51 & $29 \cdot 17$ & $8 \cdot 03$ & $6 \cdot 13$ \\
\hline Sesamolin & $7 \cdot 48$ & $8 \cdot 44$ & - & $12 \cdot 40$ & $16 \cdot 49$ & $13 \cdot 40$ & 3.64 & $2 \cdot 32$ \\
\hline Total lignans & $32 \cdot 16$ & $31 \cdot 01$ & 0.33 & $55 \cdot 86$ & $62 \cdot 01$ & $43 \cdot 72$ & $23 \cdot 25$ & $12 \cdot 26$ \\
\hline
\end{tabular}

*For experiment 1 , intake per animal corresponds to the single dose; for experiment 2 , intake is per animal per $\mathrm{d}$.

† Seeds: raw dehulled sesame seeds-commercial feed, 80:20.

†Tahini: tahini-commercial feed, 65:35.

$\S$ Control: commercial feed.

|| Seeds: raw dehulled sesame seeds-commercial feed, 30:70.

I Tahini: tahini-commercial feed, 30:70.

** Oil: sesame oil-commercial feed, 15:85.

†† Perisperm: sesame seed perisperm-commercial feed, 20:80.

㧊 Extract: polyphenolic extract-commercial feed, 0.1:99.9. 

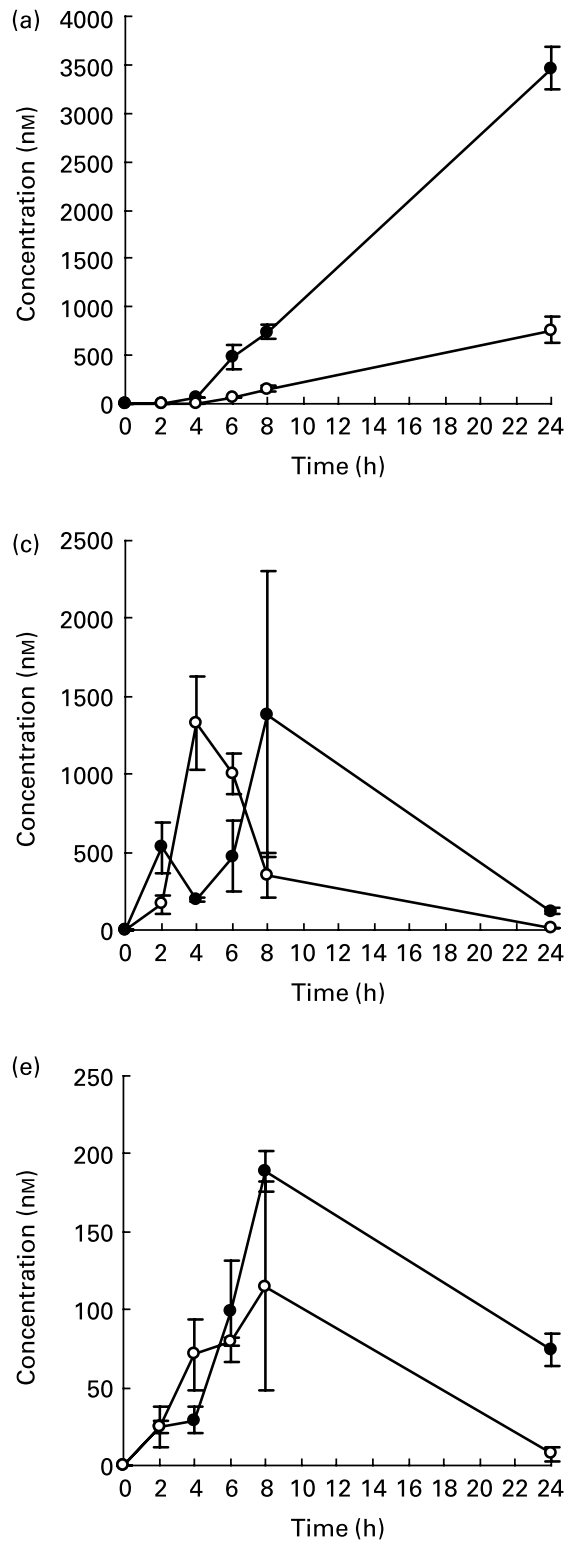
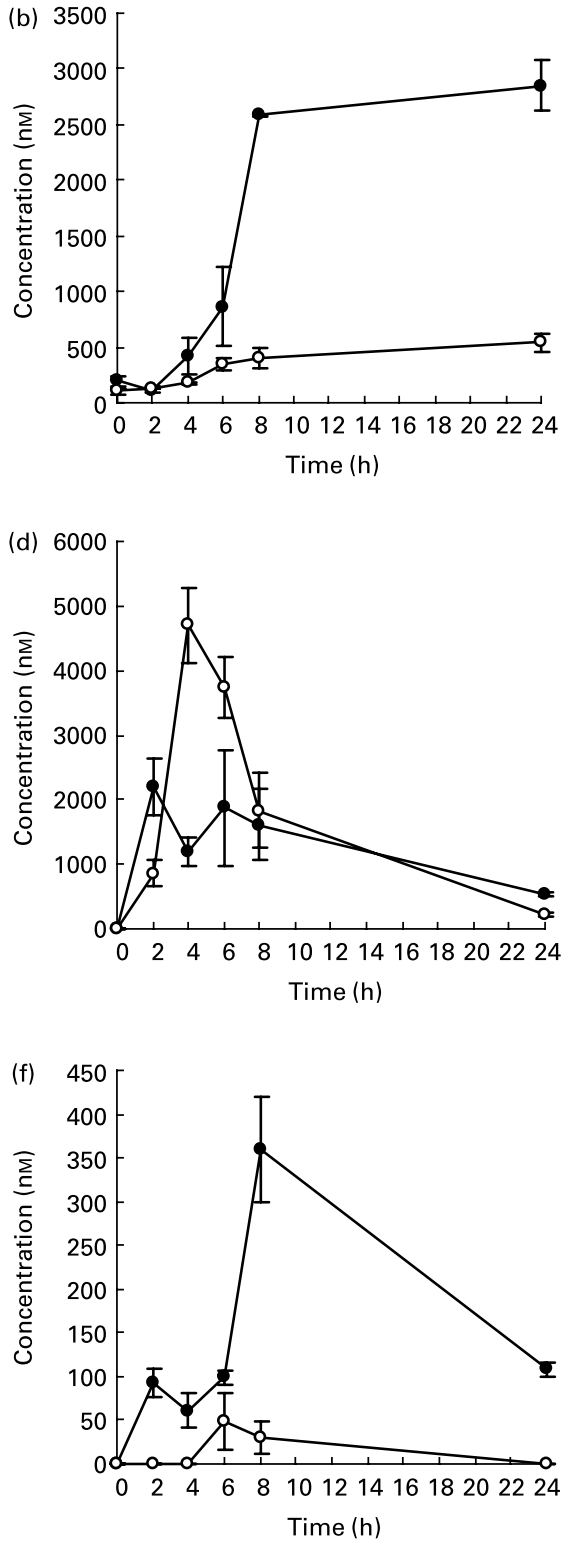

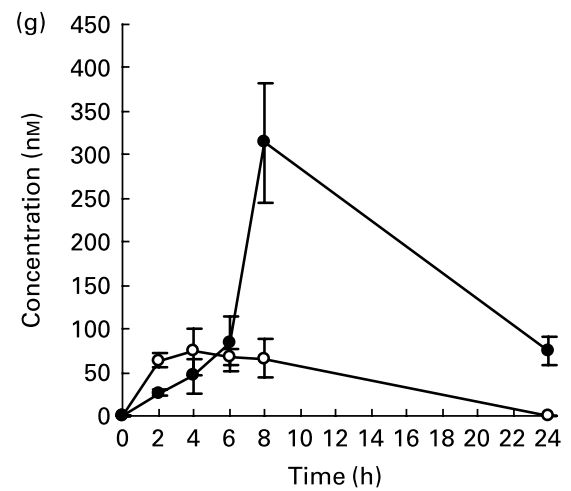

Fig. 1. Concentration (nM) of the lignans identified in the plasma of experimental animals at different time intervals after a single administration of experimenta diets based on either tahini (--) or raw dehulled sesame seeds (-O-). (a) Enterodiol; (b) enterolactone; (c) sesamin; (d) sesamolin; (e) pinoresinol; (f) sesaminol; $(\mathrm{g})$ lariciresinol. Values are means, with standard errors represented by vertical bars. 
Among the sesame lignans, sesamolin reached the highest peak concentration in both the raw seeds and the tahini groups. In the group provided with raw seeds, peak concentration was recorded $4 \mathrm{~h}$ after administration, whereas in the tahini group peak concentration was recorded in just $2 \mathrm{~h}$, maintaining a more or less stable value until $8 \mathrm{~h}$ after administration.

Sesamin concentration also reached high peak values in both groups, although not as high as sesamolin. Peak concentration values were recorded 4 and $6 \mathrm{~h}$ after administration for the raw seeds and the tahini groups respectively.

Relatively low amounts of lariciresinol, pinoresinol and sesaminol were detected, reflecting their low concentration in sesame seeds. In the tahini group, the concentrations for all lignans were higher than in the raw seeds-fed group. Peak concentrations were detected $6-8 \mathrm{~h}$ after administration in both groups.

In hour 1 of the experiment, the enterodiol and enterolactone concentration values were low, as expected. Thereafter, a gradual increase in their concentration was observed during the course of the experiment. For both compounds, peak values were reached $24 \mathrm{~h}$ after administration of the experimental diets, with the tahini group exhibiting higher concentration than the raw seeds group.

\section{Extended feeding (experiment 2)}

A significant increase of enterodiol and enterolactone concentration was observed in the blood plasma for all experimental diet groups (Table 3). The highest enterodiol levels were observed in the raw seeds and tahini groups, whereas the highest enterolactone concentration was observed in the perisperm group. Enterolactone was the predominant enterolignan in all groups but two (raw seeds and tahini). Lariciresinol and sesamin were detected in low amounts in all experimental groups with the exception of sesamin in the perisperm group, where the amount was found to be high. Pinoresinol and sesaminol where not detected. Sesamolin was the most abundant sesame lignan and it was present in all groups except the control and the polyphenolic extract groups.

\section{Discussion}

According to the data presented in the present study, ingestion of sesame-based products by experimental animals results in elevated enterodiol and enterolactone levels in blood plasma, which are maintained when a daily intake of those products is established. This is the first time, to the best of our knowledge, that sesame-based food products and by-products such as tahini, sesame oil and sesame seed perisperm have been assayed in terms of the effect they may have upon the uptake and availability of lignans in plasma. It has been reported that administration of a single dose of $25 \mathrm{~g}$ sesame seeds in human subjects results in a 50-fold increase in enterodiol and enterolactone concentration after $24 \mathrm{~h}^{(8)}$. High levels of the enterolignans were excreted in rat urine $10 \mathrm{~d}$ after feeding with a sesame-enriched $\operatorname{diet}^{(20)}$.

The concentration of sesame lignans in plasma was found to increase dramatically after a single dose of sesame seeds or tahini. This finding was more pronounced in the case of sesamin and sesamolin. They could be still detected in the plasma $24 \mathrm{~h}$ after administration, in contrast to the findings of other investigators ${ }^{(8,17)}$. Sesamin and sesamolin (along with lariciresinol) were also present in the plasma of experimental animals that received sesame product-enriched diets for 2 weeks. Sesamolin was again the most abundant lignan. There is a controversy concerning the levels of sesamolin in the plasma after administration of this lignan. Whereas according to one report $^{(15)}$, low levels of sesamolin were detected in the plasma after a period of 2 weeks' feeding with a $1 \%$ sesamolin diet, according to another high levels have been reported after administration of a diet containing $0.2 \%$ sesamolin $^{(21)}$.

As it becomes evident, according to the present results, the form of the sesame product provided through the diet influenced the levels of sesame lignans and enterolignans. Enterodiol and enterolactone concentrations were much higher in the tahini group than in the raw seeds group, after a single dose. The concentration of the sesame lignans was also higher in the tahini group, except in the case of sesamolin. However, total lignan intake was almost the same in the raw seeds and the tahini groups. We presume that the differences in lignan

Table 3. Sesame and mammalian lignans in blood plasma as determined in the extended feeding experiment (experiment 2) (Mean values with their standard errors)

\begin{tabular}{|c|c|c|c|c|c|c|c|c|c|c|c|c|}
\hline & \multicolumn{12}{|c|}{ Concentration (nmol/l) } \\
\hline & \multicolumn{2}{|c|}{ Enterodiol } & \multicolumn{2}{|c|}{ Enterolactone } & \multicolumn{2}{|c|}{$\begin{array}{l}\text { Enterodiol + } \\
\text { enterolactone }\end{array}$} & \multicolumn{2}{|c|}{ Lariciresinol } & \multicolumn{2}{|c|}{ Sesamin } & \multicolumn{2}{|c|}{ Sesamolin } \\
\hline & Mean & SEM & Mean & SEM & Mean & SEM & Mean & SEM & Mean & SEM & Mean & SEM \\
\hline Control* $^{*}$ & $0.0^{d}$ & - & $68 \cdot 5^{\mathrm{b}}$ & $6 \cdot 0$ & $68 \cdot 5^{d}$ & $6 \cdot 0$ & $0.0^{\mathrm{b}}$ & - & $0.0^{c}$ & - & $0.0^{c}$ & - \\
\hline Seeds† & $2663 \cdot 4^{a}$ & 381.5 & $1416 \cdot 7^{\mathrm{b}}$ & $197 \cdot 0$ & $4080 \cdot 1^{a}$ & $526 \cdot 6$ & $0.0^{\mathrm{b}}$ & - & $19 \cdot 6^{\mathrm{b}}$ & $2 \cdot 0$ & $154 \cdot 1^{\mathrm{b}}$ & $56 \cdot 7$ \\
\hline Perisperm $\ddagger$ & $1214 \cdot 7^{b}$ & $198 \cdot 7$ & $2133 \cdot 9^{a}$ & $408 \cdot 6$ & $3348 \cdot 6^{a}$ & 603.4 & $61 \cdot 1^{\mathrm{a}}$ & $21 \cdot 1$ & $373 \cdot 7^{a}$ & 273.5 & $721 \cdot 2^{\mathrm{a}}$ & $453 \cdot 8$ \\
\hline Tahini§̧ & $2334 \cdot 9^{a}$ & $218 \cdot 1$ & $1133 \cdot 0^{\mathrm{b}, \mathrm{c}}$ & $102 \cdot 0$ & $3467.9^{a}$ & 173.9 & $41 \cdot 1^{\mathrm{a}}$ & 3.9 & $37 \cdot 5^{b}$ & 11.0 & $697 \cdot 8^{a}$ & $116 \cdot 4$ \\
\hline Oill| & $33 \cdot 1^{\mathrm{c}}$ & 4.0 & $824 \cdot 4^{\mathrm{C}}$ & 84.4 & $844.9^{b}$ & 83.5 & $36 \cdot 7^{a}$ & $1 \cdot 1$ & $0.0^{\mathrm{c}}$ & - & $62 \cdot 7^{\mathrm{b}}$ & $12 \cdot 2$ \\
\hline Extract $\emptyset$ & $0.0^{d}$ & - & $460 \cdot 6^{d}$ & 41.6 & $460 \cdot 6^{c}$ & 41.6 & $41 \cdot 5^{a}$ & 8.5 & $19 \cdot 6^{b}$ & $2 \cdot 7$ & $0.0^{c}$ & - \\
\hline
\end{tabular}

a,b,c,d Values within a column with unlike superscript letters were significantly different $(P<0.05)$.

*Control: commercial feed.

† Seeds: raw dehulled sesame seeds-commercial feed, 30:70.

† Perisperm: sesame seed perisperm-commercial feed, 20:80.

$\S$ Tahini: tahini-commercial feed, 30:70.

\| Oil: sesame oil-commercial feed, 15:85

- Extract: polyphenolic extract-commercial feed, 0.1:99.9. 
concentrations between the raw seeds and the tahini groups are the result of both the higher absorption and the higher microbial transformation of the sesame lignans to enterodiol and enterolactone. It has been reported that milling and crushing of flaxseed increases the levels of enterolignans compared with the whole flaxseed due to improved accessibility of colonic bacteria to the lignans ${ }^{(22)}$. Tahini is a paste of ground, roasted sesame seed and thus sesame lignans are more easily accessible. Worth noticing is the finding that the difference in the levels of the enterolignans between the raw seeds diet and the tahini diet is no longer evident after treating the animals with the experimental diets for 2 weeks. This could be attributed to the adaptation of the organism (the metabolic pathways and the gut microflora) to the diet.

The most interesting observation in the short-term feeding is that no significant difference was observed in plasma concentrations of enterolignans in experimental animals treated with raw seeds, tahini and perisperm, despite the fact that the total lignan intake in the perisperm group is only about $40 \%$ of the respective total intake in the other two groups. A possible explanation is that other lignans could be present in the seed perisperm, which can be metabolised to enterodiol and enterolactone. Dietary fibre could also be responsible for the high enterolignan concentrations. The sesame seed coat has a high percentage of total fibres ${ }^{(10)}$, and it has been reported that a diet rich in fibre results in elevated levels of enterolignans, probably through modification and/or stimulation of colon microbiota ${ }^{(6,23)}$.

The diet enriched with the polyphenolic extract exhibited the lowest plasma concentrations of enterodiol and enterolactone among all the experimental groups, though significantly higher than the control group. That observation is in accordance with the low lignan intake of the polyphenolic extract group. Although other lignans are present in the extract ${ }^{(11)}$, they do not seem to be converted to either enterodiol and/or enterolactone.

Overall, according to the results of the present report, various forms of sesame-based diets result in differences in the concentration of lignans in plasma. Also, sesamin and sesamolin are detected in the plasma even $24 \mathrm{~h}$ after administration. Sesame seed perisperm, a material usually discarded as waste by the food industry, contains significant amounts of lignans that are very efficiently metabolised to enterolactone and enterodiol. Therefore the perisperm could be used for producing novel products. Moreover, the polyphenol-rich extract from the seed perisperm could also be used for the manufacture of a dietary supplement, thus rendering the seed perisperm a valuable material.

\section{Acknowledgements}

The present work was supported by a grant from GSRT Greece (01ED439) and the food company Haitoglou Bros SA.

E. N. P., D. L., E. P.-M. and A. I. P. worked as a team preparing and providing the diets to experimental animals, collecting and preparing the biological material, and analysing the amount of the lignans within the plasma of the experimental animals. R. G., P. M. and A. L. S. also worked as a team, isolating and identifying the lignans from the sesame seeds and perisperm, and preparing and providing the purified form of lignans that were used as reference material for the estimation of the lignans in the plasma of the experimental animals after they were treated with the certain diets.

There is no conflict of interest.

\section{References}

1. Webb AL \& McCullough ML (2005) Dietary lignans: potential role in cancer prevention. Nutr Cancer 51, 117-131.

2. Raffaelli B, Hoikkala A, Leppälä E \& Wähälä K (2002) Enterolignans. J Chromatogr B 777, 29-43.

3. Smeds IA, Eklund PC, Sjöholm RE, Willför SM, Nishibe S, Deyama T \& Holmbom BR (2007) Quantification of a broad spectrum of lignans in cereals, oilseeds, and nuts. J Agric Food Chem 55, 1337-1346.

4. Pietinen P, Stumpf K, Männistö S, Kataja V, Uusitupa M \& Adlercreutz H (2001) Serum enterolactone and risk of breast cancer: a case-control study in Eastern Finland. Cancer Epidemiol Biomarkers Prev 10, 339-344.

5. Nesbitt PD, Lam Y \& Thompson LU (1999) Human metabolism of mammalian lignan precursors in raw and processed flaxseed. Am J Clin Nutr 69, 549-555.

6. Nicolle C, Manach C, Morand C, Mazur W, Adlercreutz H, Rémésy C \& Scalbert A (2002) Mammalian lignan formation in rats fed a wheat bran diet. J Agric Food Chem 50, $6222-6226$.

7. Heinonen $S$, Nurmi $T$, Liukkonen $K$, Poutanen $K$, Wähälä K, Deyama T, Nishibe S \& Adlercreutz H (2001) In vitro metabolism of plant lignans: new precutsors of mammalian lignans enterolactone and enterodiol. J Agric Food Chem 49, 3178-3186.

8. Peñalvo JL, Heinonen S-M, Aura A-M \& Adlercreutz H (2005) Dietary sesamin is converted to enterolactone in humans. J Nutr 135, 1056-1062.

9. Hedelin M, Klint A, Chang ET, et al. (2006) Dietary phytoestrogen, serum enterolactone and risk of prostate cancer: the Cancer Prostate Sweden Study (Sweden). Cancer Causes Control 17, 169-180.

10. Elleuch M, Besbes S, Roiseux O, Blecker C \& Attia H (2007) Quality characteristics of sesame seeds and by-products. Food Chem 103, 641-650.

11. Grougnet R, Magiatis P, Mitaku S, Terzis A, Tillequin F \& Skaltsounis A-L (2006) New lignans from the perisperm of Sesamum indicum. J Agric Food Chem 54, 7570-7574.

12. Namiki M (1995) The chemistry and physiological functions of sesame. Food Rev Int 11, 281-329.

13. Kamal-Eldin A, Pettersson D \& Appelqvist LA (1995) Sesamin (a compound from sesame oil) increases tocopherol levels in rats fed ad libitum. Lipids 30, 499-505.

14. Sugano M, Inoue T, Koba K, Yoshida K, Hirose N, Shinmen Y, Akimoto K \& Amachi T (1990) Influence of sesame lignans on various lipid parameters in rats. Agric Biol Chem 54, $2669-2673$.

15. Kang M-H, Naito M, Tsujihara N \& Osawa T (1998) Sesamolin inhibits lipid peroxidation in rat liver and kidney. J Nutr 128, $1018-1022$.

16. Kushiro M, Takahashi K \& Ide T (2004) Species differences in the physiological activity of dietary lignan (sesamin and episesamin) in affecting hepatic fatty acid metabolism. Br J Nutr 91, 377-386.

17. Umeda-Sawada R, Ogawa M \& Igarashi O (1999) The metabolism and distribution of sesame lignans (sesamin and episesamin) in rats. Lipids 34, 633-637.

18. Hirose $\mathrm{N}$, Inoue $\mathrm{T}$, Nishihara $\mathrm{K}$, Sugano $\mathrm{M}$, Akimoto $\mathrm{K}$, Shimizu S \& Yamada H (1991) Inhibition of cholesterol absorption and synthesis in rats by sesamin. J Lipid Res 32, 629-638. 
19. Fukuda Y, Nagata M, Osawa T \& Namiki M (1986) Contribution of lignan analogues to antioxidative activity of refined unroasted sesame seed oil. J Am Oil Chem Soc 63, 1027-1031.

20. Liu Z, Saarinen NM \& Thompson LU (2006) Sesamin is one of the major precursors of mammalian lignans in sesame seed (Sesamum indicum) as observed in vitro and in rats. $J$ Nutr 136, 906-912.

21. Lim JS, Adachi Y, Takahashi Y \& Ide T (2007) Comparative analysis of sesame lignans (sesamin and sesamolin) in affecting hepatic fatty acid metabolism in rats. Br J Nutr 97, 85-95.
22. Kuijsten A, Arts ICW, van't Veer P \& Hollman PCH (2005) The relative bioavailability of enterolignans in humans is enhanced by milling and crushing of flaxseed. $J$ Nutr 135, 2812-2816.

23. Aura A-M, Oikarinen S, Mutanen M, Heinonen S-M, Adlercreutz HCT, Virtanen H \& Poutanen K (2006) Suitability of a batch in vitro fermentation model using human faecal microbiota for prediction of conversion of flaxseed lignans to enterolactone with reference to an in vivo rat model. Eur J Nutr 45, $45-51$. 